\title{
Wormhole Solutions in the Presence of Nonlinear Maxwell Field
}

\author{
S. H. Hendi ${ }^{1,2}$ \\ ${ }^{1}$ Physics Department and Biruni Observatory, College of Sciences, Shiraz University, Shiraz 71454, Iran \\ ${ }^{2}$ Research Institute for Astronomy and Astrophysics of Maragha (RIAAM), P.O. Box 55134-441, Maragha, Iran
}

Correspondence should be addressed to S. H. Hendi; hendi@shirazu.ac.ir

Received 10 September 2013; Accepted 21 February 2014; Published 26 March 2014

Academic Editor: Ian Jack

Copyright (c) 2014 S. H. Hendi. This is an open access article distributed under the Creative Commons Attribution License, which permits unrestricted use, distribution, and reproduction in any medium, provided the original work is properly cited. The publication of this article was funded by SCOAP ${ }^{3}$.

In generalizing the Maxwell field to nonlinear electrodynamics, we look for the magnetic solutions. We consider a suitable real metric with a lower bound on the radial coordinate and investigate the properties of the solutions. We find that in order to have a finite electromagnetic field near the lower bound, we should replace the Born-Infeld theory with another nonlinear electrodynamics theory. Also, we use the cut-and-paste method to construct wormhole structure. We generalize the static solutions to rotating spacetime and obtain conserved quantities.

\section{Introduction}

A wormhole can be defined as a tunnel which can join two universes [1-3]. Since General Relativity does not preclude the existence of (traversable) wormholes, a large number of papers have been written which clarify, support, or contradict much of the research about wormholes.

Morris et al. [1-3] have shown that in order to construct a traversable wormhole, one needs to have extraordinary material, denoted as exotic matter. Exotic matter can guarantee the flare-out condition of the wormhole at its throat. Unlike the classical form of matter [4], it is believed that the exotic matter violates the well-known energy conditions such as the null energy conditions (NEC), weak energy conditions (WEC), strong energy conditions (SEC), and dominant energy conditions (DEC). One of the open questions about the exotic matter is that if it can be formed in macroscopic quantities or not. We should note that these energy conditions are violated by certain states of quantum fields, amongst which one may refer to the Casimir energy, Hawking evaporation, and vacuum polarization [5-12]. Furthermore, it has been shown that one of the effective causes of the (late time) cosmic acceleration is an exotic fluid [13-16]. Hence, one is motivated to study wormhole solutions, at least geometrically.

Many authors have extensively considered the nonlinear electrodynamics and used their results to explain some physical phenomena [17-55]. A charged system whose performance cannot be described by the linear equations may be characterized with nonlinear electrodynamics. From mathematical point of view, since Maxwell equations originated from the empirical nature, we can consider a general nonlinear theory of electrodynamics and state that the Maxwell fields are only approximations of nonlinear electrodynamics, where these approximations break down for the small distances. From physical viewpoint, generalizations of Maxwell theory to nonlinear electrodynamics were introduced to eliminate infinite quantities in theoretical analysis of the electrodynamics [29-40]. In addition, one may find various limitations of the linear electrodynamics in $[56,57]$.

Recently, we have taken into account new classes of nonlinear electrodynamics, such as Born-Infeld- (BI-) like [5355] and power-Maxwell invariant (PMI) [41-52] nonlinear electrodynamics, in order to obtain new analytical solutions in Einstein and higher derivative gravity. Traversable wormholes in the Dvali-Gabadadze-Porrati theory with cylindrical symmetry have been studied in [58]. Higher dimensional Lorentzian wormholes have been analyzed by several authors [59-61]. Moreover, wormhole solutions of higher derivative gravity with linear and nonlinear Maxwell fields have been considered in [62-66]. For other kinds of wormhole solutions, we refer the reader to [62-83] and references therein. 
Motivated by the above considerations, in this paper we look for the analytical magnetic horizonless solutions of Einstein gravity with nonlinear Maxwell source. Properties of the solution will be investigated.

\section{Field Equations and Wormhole Solutions}

The field equations of Einstein gravity with an arbitrary $U(1)$ gauge field as a source may be written as

$$
\begin{gathered}
G_{\mu \nu}+\Lambda g_{\mu \nu}=\frac{1}{2} g_{\mu \nu} L(\mathscr{F})-2 L_{\mathscr{F}} F_{\mu \lambda} F_{\nu}^{\lambda}, \\
\partial_{\mu}\left(\sqrt{-g} L_{\mathscr{F}} F^{\mu \nu}\right)=0,
\end{gathered}
$$

where $G_{\mu \nu}$ is the Einstein tensor, $\Lambda=-3 / 2 l^{2}$ denotes the fourdimensional negative cosmological constant, and $L(\mathscr{F})$ is an arbitrary function of the closed 2-form Maxwell invariant $\mathscr{F}=F_{\mu \nu} F^{\mu \nu}$ and $L_{\mathscr{F}}=d L(\mathscr{F}) / d \mathscr{F}$

In addition to PMI and BI theories, in this paper, we take into account the recently proposed BI-like models [53-55], which we called as exponential form of nonlinear electrodynamics theory (ENE) and logarithmic form of nonlinear electrodynamics theory (LNE), whose Lagrangians are

$$
L(\mathscr{F})= \begin{cases}-\mathscr{F}^{s}, & \text { PMI } \\ 4 \beta^{2}\left(1-\sqrt{1+\frac{\mathscr{F}}{2 \beta^{2}}}\right), & \text { BI } \\ \beta^{2}\left(\exp \left(-\frac{\mathscr{F}}{\beta^{2}}\right)-1\right), & \text { ENE } \\ -8 \beta^{2} \ln \left(1+\frac{\mathscr{F}}{8 \beta^{2}}\right), & \text { LNE, }\end{cases}
$$

where $s$ and $\beta$ are two nonlinearity parameters. Expanding the mentioned Lagrangians near the linear Maxwell case $(s \rightarrow 1$ and $\beta \rightarrow \infty)$, one can obtain

$$
\begin{aligned}
& L(\mathscr{F}) \\
& \longrightarrow L_{\mathrm{Max}}+ \begin{cases}-\mathscr{F} \ln (\mathscr{F})(s-1)+O(s-1)^{2}, & \text { PMI } \\
+\frac{\chi \mathscr{F}^{2}}{16 \beta^{2}}+O\left(\frac{\mathscr{F}^{3}}{\beta^{4}}\right), & \text { others }\end{cases}
\end{aligned}
$$

where Maxwell Lagrangian $L_{\mathrm{Max}}=-\mathscr{F}$ and $\chi=1,2$, and 8 for LNE, BI, and ENE branches, respectively.

Investigation of the effects of the higher derivative corrections to the gauge field seems to be an interesting phenomenon. These nonlinear electrodynamics sources have different effects on the physical properties of the solutions. For example, in black hole framework, these nonlinearities may change the electric potential, temperature, horizon geometry, energy density distribution, and also asymptotic behavior of the solutions. In what follows, we study the effects of nonlinearity on the magnetic solutions.
Motivated by the fact that we are looking for the horizonless magnetic solution (instead of electric one), one can start with the following 4-dimensional spacetime:

$$
d s^{2}=-\frac{r^{2}}{l^{2}} d t^{2}+\frac{d r^{2}}{f(r)}+\Upsilon^{2} l^{2} f(r) d \theta^{2}+r^{2} d \phi^{2}
$$

where $\Upsilon$ is a constant and will be fixed later. We should note that because of the periodic nature of $\theta$, one can obtain the presented metric (5) with local transformations $t \rightarrow i l \Upsilon \theta$ and $\theta \rightarrow i t / l$ in the Schwarzschild metric with zero curvature boundary, $d s^{2}=-f(r) d t^{2}+d r^{2} / f(r)+r^{2}\left(d \theta^{2}+d \phi^{2}\right)$. In other words, metric (5) may be locally mapped to Schwarzschild spacetime but not globally. Considering the mentioned local transformation, one can find that the nonzero component of the gauge potential is $A_{\theta}$ :

$$
A_{\mu}=h(r) \delta_{\mu}^{\theta}
$$

where $h(r)$ is an arbitrary function of $r$. Using (2) with the metric (5), we find $h(r)=\int E(r) d r$ in which

$$
E(r)= \begin{cases}\frac{2 q l^{2} \Upsilon^{2}}{r^{2 /(2 s-1)},} & \text { PMI } \\ \frac{2 q l^{2} \Upsilon^{2}}{\Gamma r^{2}}, & \text { BI } \\ \frac{2 q l^{2} \Upsilon^{2}}{r^{2}} \exp \left(-\frac{L_{W}}{2}\right), & \text { ENE } \\ \frac{\beta^{2} r^{2}(1-\Gamma)}{q}, & \text { LNE, }\end{cases}
$$

where $L_{W}=\operatorname{Lambert} W(X), X=-16 q^{2} l^{2} \Upsilon^{2} / \beta^{2} r^{4}$, and $\Gamma=\sqrt{1+X / 4}$ and therefore the nonzero component of electromagnetic field tensor is

$$
F_{r \theta}=E(r)
$$

We should note that the physical gauge potential should vanish for large values of $r$. This condition is satisfied for $1 / 2<s<3 / 2$ and arbitrary $\beta$ (the mentioned constraint for $s$ is used throughout the rest of the paper). In addition, in order to have real solutions, one should restrict the radial coordinate of $\mathrm{BI}$ and LNE branches to $r \geq r_{0}$ in which

$$
r_{0}= \begin{cases}0, & \text { PMI } \\ \sqrt{\frac{2 q l \Upsilon}{\beta}}, & \text { BI } \\ 2 \sqrt{\frac{q l \Upsilon}{\beta}} \exp (1), & \text { ENE } \\ \sqrt{\frac{2 q l \Upsilon}{\beta}}, & \text { LNE. }\end{cases}
$$




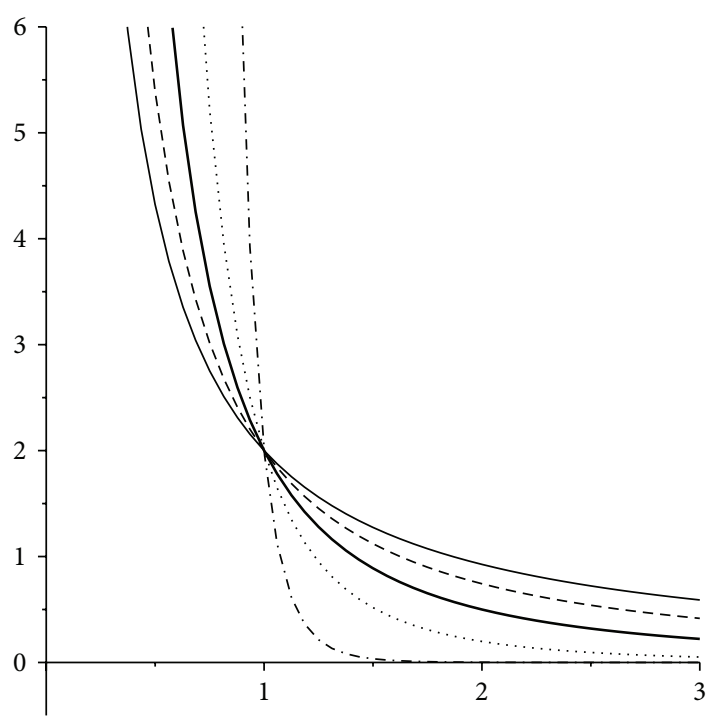

FIGURE 1: $E(r)$ versus $r$ for $q=1, \Upsilon=1, l=1$, and $s=1.4$ (solid line), $s=1.2$ (dashed line), $s=1$ "Maxwell field" (bold line), $s=0.8$ (dotted line), and $s=0.6$ (dash-dotted line).

Now, one can expand (7) to obtain the leading nonlinearity correction of Maxwell field as follows:

$$
\begin{aligned}
& \left.E(r)\right|_{\text {near the linear case }} \\
& =\frac{2 q l^{2} \Upsilon^{2}}{r^{2}}+ \begin{cases}\frac{8 q l^{2} \Upsilon^{2} \ln (r)}{r^{2}}(s-1)+O(s-1)^{2}, & \text { PMI } \\
\frac{2 \chi q^{3} l^{4} \Upsilon^{4}}{\beta^{2} r^{6}}+O\left(\frac{1}{\beta^{4}}\right) . & \text { others. }\end{cases}
\end{aligned}
$$

In addition, we can investigate the behavior of electromagnetic fields near the lower bound $r_{0}$ as

$$
\lim _{r \rightarrow r_{0}^{+}} E(r)= \begin{cases}+\infty, & \text { PMI } \\ +\infty, & \text { BI } \\ \frac{\beta \Upsilon l}{2}, & \text { ENE } \\ 2 \beta \Upsilon l, & \text { LNE. }\end{cases}
$$

In order to examine the effect of nonlinearity on the electromagnetic field, we plot Figures 1 and 2. Figure 1 shows that when we reduce the nonlinearity $s$, the electromagnetic field of the PMI branch diverges for $r \rightarrow 0$ more rapidly and for large distances it goes to zero more quickly. Figure 2 shows that for all BI-like branches, the electromagnetic field (the same behavior as in Maxwell case) vanishes for large $r$. Moreover, in addition to (11), Figure 2 shows that near the lower bound $\left(r_{0}\right)$, unlike BI branch, the electromagnetic fields of LNE and ENE branches have finite values. In other words, considering the LNE and ENE branches, we could remove the lower bound divergency of electromagnetic field.

Taking into account the electromagnetic field tensor, we are in a position to find the function $f(r)$. In order to obtain

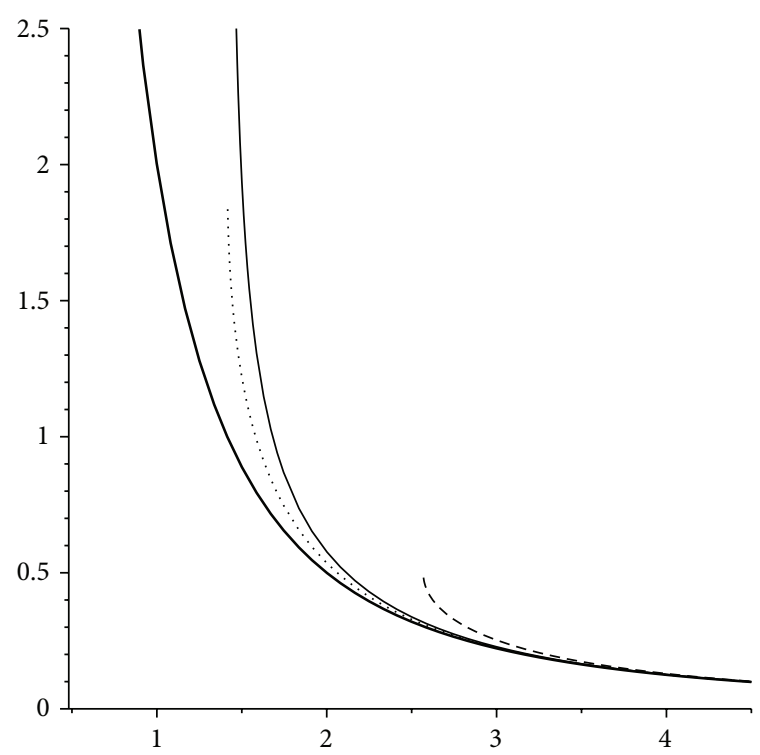

FIGURE 2: $E(r)$ versus $r$ for $q=1, \Upsilon=1, l=1$, and $\beta=1$. BI (solid line), ENE (dashed line), LNE (dotted line), and Maxwell field (bold line).

it, one may use any components of (1). We simplify the components of (1) and find that the nonzero independent components of (1) are

$$
\begin{gathered}
f^{\prime \prime}(r)+\frac{2 f^{\prime}(r)}{r}+2 \Lambda+\Delta_{1}(r)=0, \\
f^{\prime}(r)+\frac{f(r)}{r}+\Lambda r+\Delta_{2}(r)=0,
\end{gathered}
$$

with

$$
\Delta_{1}(r)= \begin{cases}-\left(\frac{2 h^{\prime 2}(r)}{\Upsilon^{2} l^{2}}\right)^{s}, & \text { PMI } \\ 4 \beta^{2}\left[\sqrt{1+\frac{h^{\prime 2}(r)}{\beta^{2} \Upsilon^{2} l^{2}}}-1\right], & \text { BI } \\ \beta^{2}\left[1-\exp \left(\frac{-2 h^{\prime 2}(r)}{\beta^{2} \Upsilon^{2} l^{2}}\right)\right], & \text { ENE } \\ 8 \beta^{2} \ln \left[1+\left(\frac{h^{\prime}(r)}{2 \beta \Upsilon l}\right)^{2}\right], & \text { LNE, }\end{cases}
$$

$\Delta_{2}(r)$

$$
= \begin{cases}\frac{r}{2}(2 s-1)\left(\frac{2 h^{\prime 2}(r)}{\Upsilon^{2} l^{2}}\right)^{s}, & \text { PMI } \\ 2 r \beta^{2}\left[\left(1+\frac{h^{\prime 2}(r)}{\beta^{2} \Upsilon^{2} l^{2}}\right)^{-1 / 2}-1\right], & \text { BI } \\ \frac{r \beta^{2}}{2}\left\{1-\left[1+\left(\frac{2 h^{\prime}(r)}{\beta \Upsilon l}\right)^{2}\right] \exp \left(\frac{-2 h^{\prime 2}(r)}{\beta^{2} \Upsilon^{2} l^{2}}\right)\right\}, & \text { ENE } \\ 4 r \beta^{2}\left\{\ln \left[1+\left(\frac{h^{\prime}(r)}{2 \beta \Upsilon l}\right)^{2}\right]-\frac{2}{1+\left(2 \beta \Upsilon l / h^{\prime}(r)\right)^{2}}\right\}, & \text { LNE, }\end{cases}
$$


where prime and double prime are first and second derivatives with respect to $r$, respectively. After some cumbersome manipulation, the solutions of (12) can be written as

$$
\begin{aligned}
& f(r) \\
& =\frac{-2 m}{r}-\frac{\Lambda r^{2}}{3} \\
& + \begin{cases}\frac{r^{2}(2 s-1)^{2}}{2(2 s-3)}\left(\frac{8 q^{2} l^{2} \Upsilon^{2}}{r^{4 /(2 s-1)}}\right)^{s}, & \text { PMI } \\
\frac{2 \beta^{2} r^{2}}{3}-\frac{2 \beta^{2}}{r} \int \Gamma r^{2} d r, & \text { BI } \\
-\frac{\beta^{2} r^{2}}{6}-\frac{2 \beta q l \Upsilon}{r} \int\left(\frac{1}{\sqrt{-L_{W}}}-\sqrt{-L_{W}}\right) d r, & \text { ENE } \\
-\frac{4 \beta^{2}}{r} \int r^{2} \ln \left(\frac{\beta^{2} r^{4}(1-\Gamma)}{2 q^{2} \Upsilon^{2} l^{2}}\right) d r+\frac{4 \beta^{2}}{r} \int r^{2}(1-\Gamma) d r, & \text { LNE, }\end{cases}
\end{aligned}
$$

where $m$ is the integration constant which is related to the mass parameter. In order to investigate the effect of nonlinearity on the metric function, simplistically, we expand $f(r)$ for $s \rightarrow 1$ for PMI and $\beta \rightarrow \infty$ for other branches. After some manipulation, we obtain

$$
\begin{aligned}
& f(r) \\
& =f_{\text {Max }}(r) \\
& + \begin{cases}-\frac{4 q^{2} l^{2} \Upsilon^{2}\left[6+\ln \left(8 q^{2} \Upsilon^{2} l^{2} r^{4}\right)\right]}{r^{2}}(s-1)+O(s-1)^{2}, & \text { PMI } \\
-\frac{2 \chi q^{4} l^{4} \Upsilon^{4}}{5 \beta^{2} r^{6}}+O\left(\frac{1}{\beta^{4}}\right), & \text { others, }\end{cases}
\end{aligned}
$$

where $f_{\mathrm{Max}}(r)$ is the magnetic solution of Einstein-Maxwell gravity:

$$
f_{\mathrm{Max}}(r)=\frac{-2 m}{r}-\frac{\Lambda r^{2}}{3}-\frac{4 q^{2} l^{2} \Upsilon^{2}}{r^{2}},
$$

and the second term on the right hand side of (15) is the leading nonlinearity correction to the Einstein-Maxwell gravity solution.

2.1. Properties of the Solutions. At first step, we should note that the presented solutions are asymptotically anti-de Sitter (adS) and they reduce to asymptotically adS EinsteinMaxwell solutions for $s \rightarrow 1$ (PMI branch) or $\beta \rightarrow \infty$ (other branches).

The second step should be devoted to looking for the singularities and hence we should calculate the curvature invariants. One can show that, for the metric (5), the Kretschmann and Ricci scalars are

$$
\begin{gathered}
R_{\mu \nu \rho \sigma} R^{\mu \nu \rho \sigma}=f^{\prime \prime 2}(r)+\frac{4 f^{\prime 2}(r)}{r^{2}}+\frac{4 f^{2}(r)}{r^{4}}, \\
R=-f^{\prime \prime}(r)-\frac{4 f^{\prime}(r)}{r}-\frac{2 f(r)}{r^{2}} .
\end{gathered}
$$

Inserting (14) into (17) and using numerical calculations, one can show that the Ricci and Kretschmann scalars diverge at $r=r_{0}$ and are finite for $r>r_{0}$ and for $r \rightarrow \infty$ one obtains

$$
\begin{gathered}
\left.R_{\mu \nu \rho \sigma} R^{\mu \nu \rho \sigma}\right|_{\text {Large } r}=\frac{8 \Lambda^{2}}{3}+\left\{\begin{array}{l}
O\left(\frac{1}{r^{\xi}}\right) \quad \text { PMI } \\
O\left(\frac{1}{r^{6}}\right), \text { others, }
\end{array}\right. \\
\left.R\right|_{\text {Large } r}=4 \Lambda+\left\{\begin{array}{l}
O\left(\frac{1}{r^{\xi}}\right) \quad \text { PMI } \\
O\left(\frac{1}{r^{8}}\right), \text { others, } \\
\xi \in(3, \infty),
\end{array}\right.
\end{gathered}
$$

which confirms that the asymptotic behavior of the solutions is adS. Considering the divergency of the Ricci and Kretschmann scalars at $r_{0}$, one may think that there is a curvature singularity located at $r=r_{0}$. This singularity will be naked if the function $f(r)$ has no real root larger than $r_{0}$ (singularity is not covered with a horizon) and we are not interested in it. Therefore, we consider the case in which the function $f(r)$ has at least a nonextreme positive real root larger than $r_{0}$. It is notable that the function $f(r)$ is negative for $r=r_{+}-\epsilon$ ( $\epsilon$ is an infinitesimal number) and positive for $r>r_{+}$where $r_{+}$is the largest positive real root of $f(r)=0$. Negativity of the function $f(r)$ leads to an apparent change of metric signature and it forces us to consider $r_{+} \leq r<\infty$. We should state that although the metric function $f(r)$ vanishes at $r=r_{+}$, we have $f^{\prime}\left(r=r_{+}\right)>0$. In addition, there is no curvature singularity in the range $r_{+} \leq r<\infty$. Following the procedure of [84], one may find that there is a conic singularity at $r=r_{+}$.

Removing this conical singularity by adjusting $\Upsilon=$ $1 /\left[l f^{\prime}\left(r_{+}\right)\right][84]$, we desire to interpret the obtained solutions as wormholes. In order to construct wormholes from the gluing, one is required to use the cut-and-paste prescription [6783]. In this method, we take into account two geodesically incomplete copies of the solutions (removing from each copy the forbidden region $\Omega$ ) with two copies of the boundaries $\partial \Omega$, where

$$
\begin{gathered}
\Omega \equiv\left\{(t, r, \theta, \phi) \mid r<r_{+}\right\}, \\
\partial \Omega \equiv\left\{(t, r, \theta, \phi) \mid r=r_{+}\right\} .
\end{gathered}
$$

Now, we identify two copies of the mentioned boundaries to build a geodesically complete manifold. This cut-and-paste method constructs a wormhole with a throat at $r=r_{+}$. In order to confirm this claim, we should check the so-called flare-out condition at the throat. To do this, one can consider a 2-dimensional submanifold of the metric (5), $d s_{2 \text {-dim }}^{2}$ (with constant $t$ and $\theta$ ), and embed it in a 3 -dimensional Euclidean flat space in cylindrical coordinates, $d s_{3-\mathrm{dim}}^{2}$, where

$$
\begin{gathered}
d s_{2-\operatorname{dim}}^{2}=\frac{d r^{2}}{f(r)}+r^{2} d \phi^{2}, \\
d s_{3-\operatorname{dim}}^{2}=d r^{2}+r^{2} d \phi^{2}+d z^{2} .
\end{gathered}
$$


Regarding the surface $z=z(r)$, we obtain

$$
\begin{gathered}
\left.\frac{d r}{d z}\right|_{r=r_{+}}=\left.\sqrt{\frac{f(r)}{1-f(r)}}\right|_{r=r_{+}}=0, \\
\left.\frac{d^{2} r}{d z^{2}}\right|_{r=r_{+}}=\left.\frac{f^{\prime}}{2[1-f]^{2}}\right|_{r=r_{+}}=\frac{1}{2} f^{\prime}\left(r=r_{+}\right)>0,
\end{gathered}
$$

which shows that the flare-out condition may be satisfied for the surface $z=z(r)$ and therefore $r=r_{+}$is the radius of the wormhole throat. It is clear to find that the metric (the first fundamental form) is continuous on the boundary $\partial \Omega$, while its first derivative may be discontinuous. In order to investigate this discontinuity, one should consider the extrinsic curvature (the second fundamental form). Here, we give a brief remark about it [85-91]. The second fundamental forms associated with the two sides of the throat $\partial \Omega$ are

$$
K_{i j}^{ \pm}=-\left.n_{\mu}^{ \pm}\left[\partial_{i} \partial_{j} x^{\mu}+\Gamma_{\alpha \beta}^{\mu} \partial_{i} x^{\alpha} \partial_{j} x^{\beta}\right]\right|_{\partial \Omega},
$$

in which $n_{\mu}^{ \pm}$are the unit normals to the two boundaries $\partial \Omega$ with the following explicit form:

$$
n_{\mu}^{ \pm}= \pm\left|\partial_{\alpha} G \partial^{\alpha} G\right|^{-1 / 2} \partial_{\mu} G
$$

where $i, j=1,2,3$ corresponding to the boundary $\partial \Omega$, $\mu, \alpha, \beta=1,2,3,4$ corresponding to original spacetime, and $G=r-r_{+}$. We emphasize that \pm sign comes from the fact that a unit's normal points outward from one side of the throat $\partial \Omega$ and points inward on the other side. Generally, we have $K_{i j}^{+} \neq K_{i j}^{-}$, and one can write the following Einstein (Lanczos) equation on the throat:

$$
k_{i j}-k g_{i j}=-8 \pi S_{i j}
$$

where $k_{i j}=K_{i j}^{+}-K_{i j}^{-}, k=k_{i}^{i}$, and $S_{i j}=\operatorname{diag}\left(\sigma, p_{\theta}, p_{\phi}\right)$ is the stress-energy tensor localized in $\partial \Omega$ with $\sigma$, the surface energy density, and $p_{\theta}$ and $p_{\phi}$, the principal surface pressures (tensions):

$$
\begin{gathered}
\sigma=-\frac{(d / d r)\left[r^{2} f(r)\right]}{8 \pi l^{2} \sqrt{f(r)}}, \\
p_{\theta}=\frac{\sqrt{f^{3}(r)} l^{2} \Upsilon^{2}}{2 \pi r}, \\
p_{\phi}=\frac{(d / d r)\left[r^{2} f(r)\right]}{8 \pi \sqrt{f(r)}} .
\end{gathered}
$$

One can use the series expansion for $r \rightarrow r_{+}$with the obtained metric function $f(r)$ to find the signs of surface energy density and tensions. Negative surface energy implies the existence of ghost-like matter at the throat and the negative signs of the tensions mean that they are indeed pressures.

Now, we should discuss the energy conditions for the wormhole solutions. On general grounds, it has been shown that traversable wormhole may exist with exotic matter which violates the null energy condition [1-3]. In order to check the energy conditions, we use the following orthonormal contravariant (hatted) basis to simplify the mathematics and physical interpretations:

$$
\begin{gathered}
\mathbf{e}_{\hat{t}}=\frac{l}{r} \frac{\partial}{\partial t}, \quad \mathbf{e}_{\hat{r}}=f^{1 / 2} \frac{\partial}{\partial r} \\
\mathbf{e}_{\hat{\theta}}=\frac{1}{\Upsilon l f^{1 / 2}} \frac{\partial}{\partial \theta}, \quad \mathbf{e}_{\hat{\phi}}=r^{-1} \frac{\partial}{\partial \phi} .
\end{gathered}
$$

Using the mentioned basis, we can obtain

$$
\begin{aligned}
& T_{\overparen{t t}}=-T_{\widehat{\phi} \hat{\phi}}= \begin{cases}\frac{1}{2}\left(\frac{8 \Upsilon^{2} q^{2} l^{2}}{r^{4 /(2 s-1)}}\right)^{s}, & \text { PMI } \\
2 \beta^{2}\left(\Gamma^{-1}-1\right), & \text { BI } \\
\frac{\beta^{2}\left(1-\sqrt{\frac{X}{L_{W}}}\right),}{2} & \text { ENE } \\
4 \beta^{2} \ln \left(\frac{-8(1-\Gamma)}{X}\right), & \text { LNE, }\end{cases} \\
& T_{\widehat{r} r}=T_{\widehat{\theta} \widehat{\theta}}= \begin{cases}\frac{(2 s-1)}{2}\left(\frac{8 \Upsilon^{2} q^{2} l^{2}}{r^{4 /(2 s-1)}}\right)^{s}, & \text { PMI } \\
2 \beta^{2}(1-\Gamma), & \text { BI } \\
\frac{\beta^{2}\left(\sqrt{\frac{X}{L_{W}}}+\sqrt{X L_{W}}-1\right),}{\beta^{2}\left[8-4 \ln \left(\frac{-8(1-\Gamma)}{X}\right)+\frac{X}{1-\Gamma}\right],} & \text { ENE }\end{cases}
\end{aligned}
$$

and therefore for $r \geq r_{+}>r_{0}$, we have

$$
T_{\overparen{t} \hat{t}}>0, \quad T_{\overparen{t t}}+T_{\widehat{r} \widehat{r}}>0,
$$

which shows that the solutions satisfy the null and weak energy conditions, simultaneously (see Figure 3 for more clarification).

At the end of this section, we desire to study the effects of the nonlinearity on energy density of the spacetime. At the start, we can expand $T_{\overparen{t} \mathfrak{t}}$ near the linear case to obtain

$$
\begin{aligned}
& T_{\tilde{t} t} \\
& =\left.T_{\tilde{t} t}\right|_{\text {Maxwell }} \\
& + \begin{cases}\frac{4 q^{2} l^{2} \Upsilon^{2} \ln \left(8 q^{2} \Upsilon^{2} l^{2} r^{4}\right)}{r^{4}}(s-1)+O(s-1)^{2}, & \text { PMI } \\
+\frac{6 \chi q^{4} l^{4} \Upsilon^{4}}{\beta^{2} r^{8}}+O\left(\frac{1}{\beta^{4}}\right), & \text { others, }\end{cases}
\end{aligned}
$$

where $\left.T_{\overparen{t} \hat{t}}\right|_{\text {Maxwell }}=4 \Upsilon^{2} q^{2} l^{2} / r^{4}$ and the second term on the right hand side of (29) is the leading nonlinearity correction to the energy density of the Einstein-Maxwell 


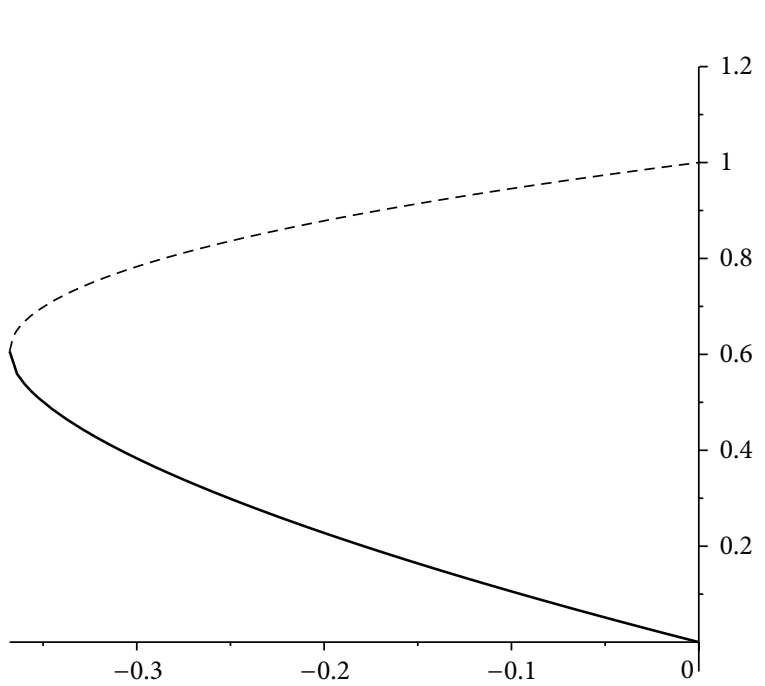

(a)

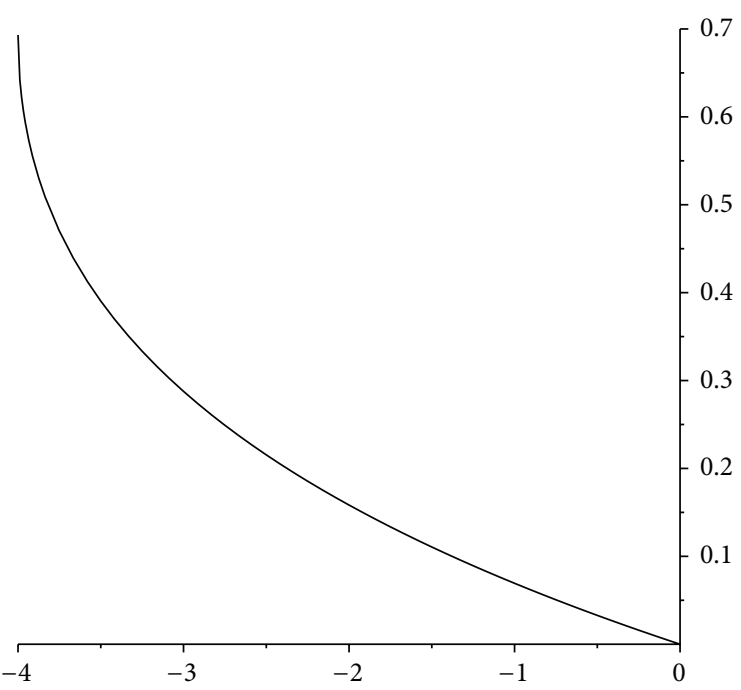

(b)

Figure 3: (a) $\sqrt{X / L_{W}}$ (dashed line) and $\sqrt{X L_{W}}$ (bold line) versus $X$. (b) $\ln (-8(\Gamma-1) / X)$ versus $X$.

theory. Investigations of the energy densities near the lower bound $r_{0}$ show that

$$
\lim _{r \rightarrow r_{0}^{+}} T_{\overparen{t t}}= \begin{cases}+\infty, & \text { PMI } \\ +\infty, & \text { BI } \\ \frac{\beta^{2}}{2}\left(1-\exp \left(-\frac{1}{2}\right)\right), & \text { ENE } \\ 4 \beta^{2} \ln 2, & \text { LNE. }\end{cases}
$$

In addition, we plot the energy density $T_{\hat{t} t}$ versus $r$ for different values of nonlinearity parameter $s$ and also various branches of BI-like fields. Figures 4 and 5 show that for the arbitrary choices of $r$ the energy density is positive definite. Furthermore, Figure 4 shows that the nonlinearity parameter, $s$, has effects on the behavior of the energy density and when we reduce $s$, both divergency of energy density near the origin and its vanishing for large values of distance occur more rapidly. Moreover, considering Figure 5 with (30), one can find that $T_{t \mathfrak{t t}}$ has a finite value for an arbitrary allowed distance in ENE and LNE branches. Like Maxwell and PMI theory, for BI branch the energy density diverges near the lower bound $r_{0}$.

2.2. Rotating Solutions. In this section, we want to add angular momentum to the static spacetime (5). To do this, one can use the following rotation boost in the $t-\theta$ plane:

$$
t \longmapsto \Xi t-a \theta, \quad \theta \longmapsto \Xi \theta-\frac{a}{l^{2}} t
$$

where $\Xi=\sqrt{1+a^{2} / l^{2}}$ and $a$ is a rotation parameter. Taking into account (31) and applying it to static metric (5), one obtains

$$
\begin{aligned}
d s^{2}= & -\frac{r^{2}}{l^{2}}(\Xi d t-a d \theta)^{2}+\frac{d r^{2}}{f(r)} \\
& +\Upsilon^{2} l^{2} f(r)\left(\frac{a}{l^{2}} d t-\Xi d \theta\right)^{2}+r^{2} d \phi^{2},
\end{aligned}
$$

where $f(r)$ is the same as $f(r)$ given in (14). It is notable that one can obtain the presented metric (32) with local transformations $t \rightarrow i l \Upsilon\left(a t / l^{2}-\Xi \theta\right)$ and $\theta \rightarrow i(\Xi t-a \theta) / l$ in the Schwarzschild metric with zero curvature boundary; $d s^{2}=-f(r) d t^{2}+d r^{2} / f(r)+r^{2} d \theta^{2}+r^{2} d \phi^{2}$. Thus, the nonzero components of the gauge potential are $A_{\theta}$ and $A_{t}$ :

$$
A_{\mu}=h(r)\left(\Xi \delta_{\mu}^{\theta}-\frac{a}{l^{2}} \delta_{\mu}^{t}\right)
$$

where $h(r)$ is the same as in the static case. Furthermore, the nonzero components of electromagnetic field tensor are given by

$$
F_{t r}=\frac{a}{\Xi l^{2}} F_{r \theta}=\frac{a}{l^{2}} E(r) .
$$

As we mentioned before, the periodic nature of $\theta$ helps us to conclude that the transformation (31) is not a proper coordinate transformation on the entire manifold and therefore the metrics (5) and (32) are distinct [92]. In addition, it is desired to note that rotating solutions have no horizon and curvature singularity. Moreover, it is worth noting that besides the magnetic field along the $\theta$ coordinate, there is also a radial electric field $\left(F_{t r} \neq 0\right)$ and, therefore, unlike the static case, the rotating wormhole has a nonzero electric charge which is proportional to the rotation parameter. 

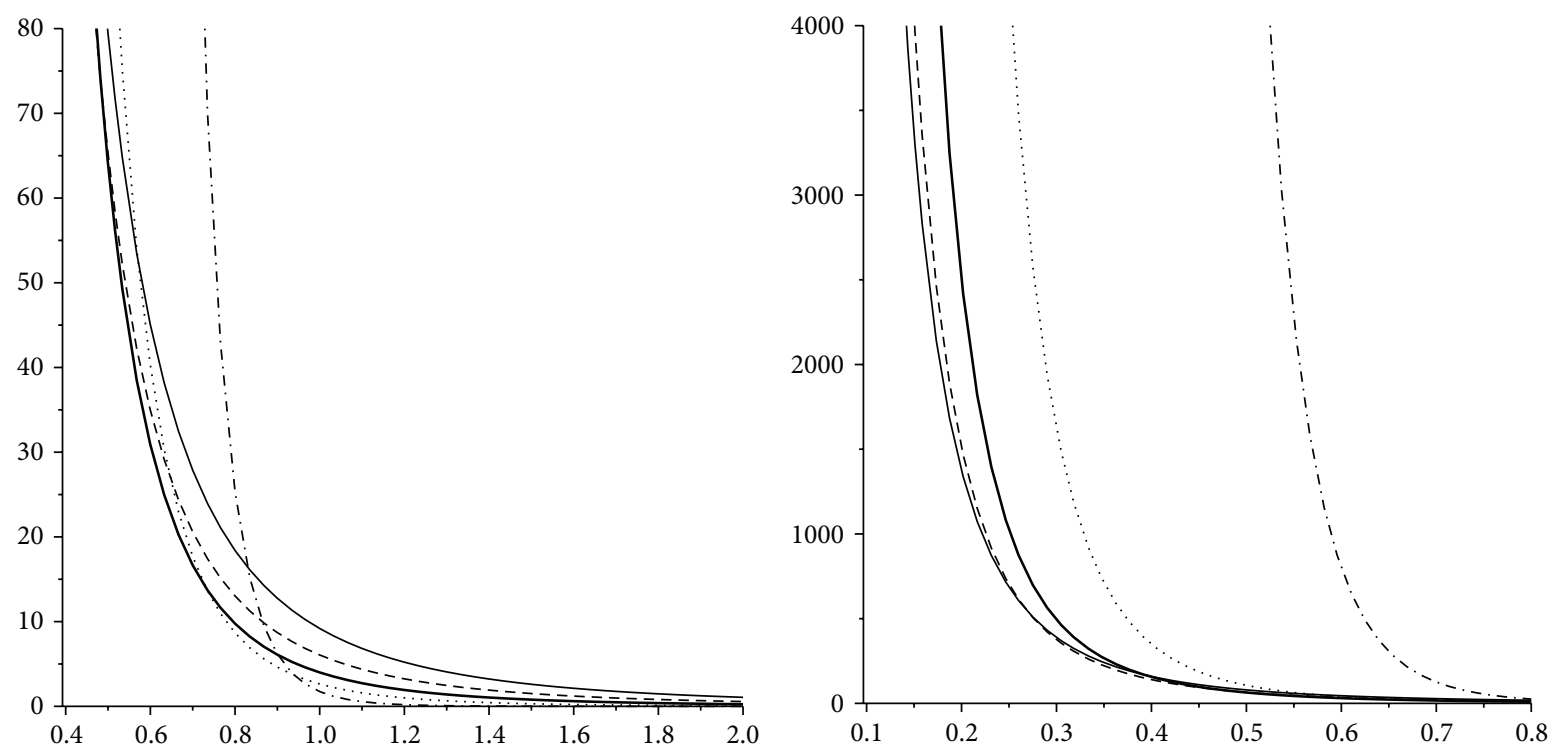

FIGURE 4: $T_{\overparen{t} \hat{t}}$ versus $r$ for $q=1, \Upsilon=1, l=1$, and $s=1.4$ (solid line), $s=1.2$ (dashed line), $s=1$ "Maxwell field" (bold line), $s=0.8$ (dotted line), and $s=0.6$ (dash-dotted line) "different scales".

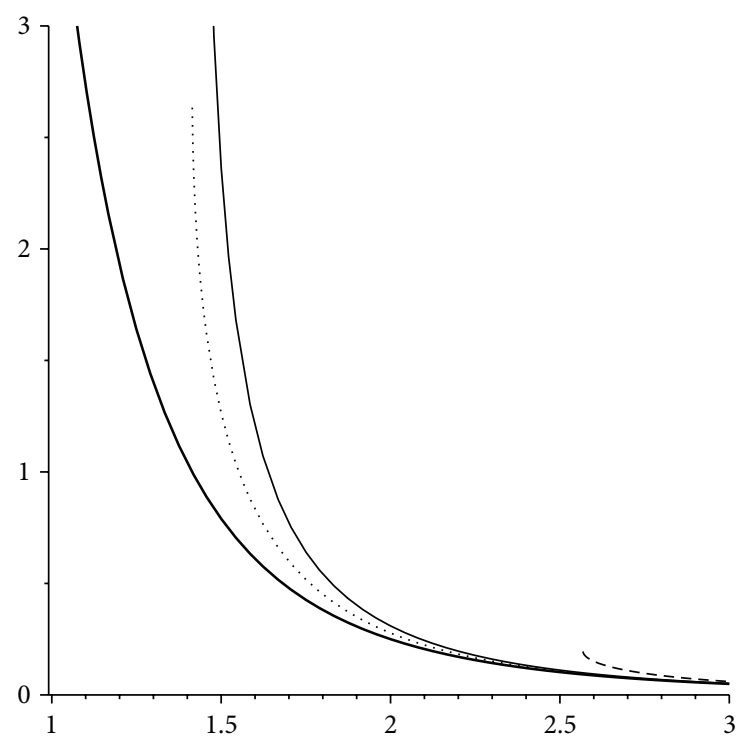

Figure 5: $T_{\overparen{t t}}$ versus $r$ for $q=1, \Upsilon=1, l=1$, and $\beta=1$. BI (solid line), ENE (dashed line), LNE (dotted line), and Maxwell field (bold line).

2.3. Conserved Quantities. Here we desire to calculate finite conserved quantities. In order to obtain a finite value for these quantities, we can use the counterterm method inspired by the concept of (AdS/CFT) correspondence [93-95]. It has been shown that for asymptotically AdS solutions the finite energy momentum tensor is

$$
T^{a b}=\frac{1}{8 \pi}\left(K^{a b}-K \gamma^{a b}-\frac{2 \gamma^{a b}}{l}\right),
$$

where $K$ is the trace of the extrinsic curvature $K^{a b}$ and $\gamma^{a b}$ is the induced metric of the boundary. Taking into account the
Killing vector field $\xi$, one may obtain the quasilocal conserved quantities in the following form:

$$
\mathcal{Q}(\xi)=\int_{\mathscr{B}} d^{2} \varphi \sqrt{\sigma} T_{a b} n^{a} \xi^{b},
$$

where $\sigma$ is the determinant of the boundary metric in ADM (Arnowitt-Deser-Misner) form $\sigma_{i j}$, and $n^{a}$ is the timelike unit vector normal to the boundary $\mathscr{B}$. Considering two Killing vectors $\xi=\partial / \partial t$ and $\zeta=\partial / \partial \theta$, we can find their associated conserved charges which are mass and angular momentum as follows:

$$
\begin{gathered}
M=4 \pi^{2}\left[3\left(\Xi^{2}-1\right)+1\right] \Upsilon m, \\
J=12 \pi^{2} \Upsilon m \Xi a,
\end{gathered}
$$

where the former equation confirms that $a$ is the rotational parameter.

Finally, we are in a position to discuss the electric charge. In order to compute it, we need a nonzero radial electric field $F_{t r}$ and therefore one expects that vanishing $F_{t r}$ (static case) leads to zero electric charge. Taking into account the Gauss law for the rotating solutions and computing the flux of the electric field at infinity, one can find

$$
Q= \begin{cases}\frac{2^{3 s+1} \pi^{2} \Upsilon s}{4 l}\left(\frac{(2 s-1) q}{(3-2 s) l}\right)^{2 s-1} a, & \text { PMI } \\ \frac{4 \pi^{2} \Upsilon q}{l^{2}} a, & \text { others }\end{cases}
$$

which confirms that the static wormholes do not have electric charge.

\section{Closing Remarks}

In this paper, we took into account a class of magnetic Einsteinian solutions in the presence of nonlinear source. 
The magnetic spacetime which we used in this paper may be obtained from the Schwarzschild metric with zero curvature boundary by the local transformations $t \rightarrow i l \Upsilon \theta$ and $\theta \rightarrow$ $i t / l$. It is notable that because of the periodic nature of $\theta$, the mentioned transformations cannot be global.

We considered four forms of nonlinear electrodynamics, namely, PMI, BI, ENE, and LNE theories, whose asymptotic behavior leads to Maxwell theory. Obtaining real solutions forced us to define a lower bound $r_{0}$ for the radial coordinate of spacetime. We investigated the effect of nonlinearity parameter on the electromagnetic field and found that, for PMI branch, if one reduces the nonlinearity parameter $s$, then the electromagnetic field diverges near the origin more rapidly and for large distances it goes to zero more quickly. In addition, we found that for all BI-like branches, the behavior of the electromagnetic field is the same as Maxwell case for large values of distance, but near the lower bound, the electromagnetic field of the ENE and LNE branches is finite and it diverges for the BI branch. It is interesting to note that the divergency of the BI branch has less strength in comparison to the the Maxwell field. Consequently, in order to have a finite electromagnetic field near the lower bound, we could not use BI theory for our magnetic spacetime and we may apply LNE and ENE theories for this purpose.

Then, we obtained the metric function for all branches and found that they reduce to asymptotically adS EinsteinMaxwell solutions for $s \rightarrow 1$ (PMI branch) or $\beta \rightarrow \infty$ (other branches). We also expanded the metric function near the linear Maxwell field and calculated the curvature scalars for large $r$ to find that obtained solutions are asymptotically anti-de Sitter (adS). Taking into account the presented metric, one can find that the function $f(r)$ cannot be negative since its negativity leads to an apparent change of metric signature. This limitation forced us to consider $r_{+} \leq r<\infty$. Using numerical calculations, one can find that there is no curvature singularity in the range $r_{+} \leq r<\infty$, but one may find a conic singularity at $r=r_{+}$.

After that, we removed the mentioned conic singularity and used the cut-and-paste prescription to construct a wormhole from the gluing and then we checked the so-called flareout condition at the throat $r=r_{+}$. Since it has been stated before that traversable wormhole may or may not exist with exotic matter [1-3], we investigated the energy conditions for the obtained wormhole solutions and found that the null and weak energy conditions are satisfied, which means that there is no exotic matter near the throat.

We also studied the effects of nonlinearity parameter on the energy density. For PMI branch, we found that when we reduce $s$, both divergency of energy density near the origin and its vanishing for large values of distance occur more rapidly. Moreover, one can find that energy density has a finite value for an arbitrary distance for LNE and ENE branches and it diverges near the lower bound for BI branch.

We generalized the static solutions to rotating ones and obtained the conserved quantities. We found that, unlike the static case, for the spinning spacetime, the wormhole has a net electric charge density. We also found that in spite of the fact that the mentioned nonlinear theories change the properties of the solutions significantly, they do not have any effect on mass and angular momentum.

\section{Conflict of Interests}

The author declares that there is no conflict of interests regarding the publication of this paper.

\section{Acknowledgments}

The author thanks the anonymous referees for the useful criticisms and comments which permitted him to improve this paper. The author is indebted to A. Poostforush and H. Mohammadpour for reading the paper. The author also wishes to thank Shiraz University Research Council. This work has been supported financially by Research Institute for Astronomy and Astrophysics of Maragha (RIAAM), Iran.

\section{References}

[1] M. S. Morris and K. S. Thorne, "Wormholes in spacetime and their use for interstellar travel: a tool for teaching general relativity," American Journal of Physics, vol. 56, no. 5, pp. 395412, 1988.

[2] M. S. Morris, K. S. Thorne, and U. Yurtsever, "Wormholes, time machines, and the weak energy condition," Physical Review Letters, vol. 61, no. 13, pp. 1446-1449, 1988.

[3] D. Hochberg and M. Visser, "Dynamic wormholes, antitrapped surfaces, and energy conditions," Physical Review D, vol. 58, no. 4, Article ID 044021, 1998.

[4] S. W. Hawking and G. F. R. Ellis, The Large Scale Structure of Space-Time, Cambridge University Press, Cambridge, UK, 1973.

[5] M. Visser, Lorentzian Wormholes: From Einstein to Hawking, AIP Press, Woodbury, NY, USA, 1995.

[6] F. S. N. Lobo, "Exotic solutions in General Relativity: traversable wormholes and warp drive spacetimes," in Classical and Quantum Gravity Research, pp. 1-78, Nova Science Publishers, 2008.

[7] C. Barceló and M. Visser, "Twilight for the energy conditions?" International Journal of Modern Physics D, vol. 11, no. 10, pp. 1553-1560, 2002.

[8] M. Visser, "Gravitational vacuum polarization. I. Energy conditions in the Hartle-Hawking vacuum," Physical Review D, vol. 54, no. 8, pp. 5103-5115, 1996.

[9] M. Visser, "Gravitational vacuum polarization. II. Energy conditions in the Boulware vacuum," Physical Review D, vol. 54, no. 8, pp. 5116-5122, 1996.

[10] M. Visser, "Gravitational vacuum polarization. III. Energy conditions in the $(1+1)$-dimensional Schwarzschild spacetime," Physical Review D, vol. 54, no. 8, pp. 5123-5128, 1996.

[11] M. Visser, "Gravitational vacuum polarization. IV. Energy conditions in the Unruh vacuum," Physical Review D, vol. 56, no. 2, pp. 936-952, 1997.

[12] M. Visser, “Gravitational vacuum polarization," http://arxiv.org/ abs/gr-qc/9710034.

[13] J. L. Tonry, B. P. Schmidt, B. Barris et al., "Cosmological Results from High-z Supernovae," The Astrophysical Journal, vol. 594, article 1, 2003.

[14] U. Alam, V. Sahni, T. D. Saini, and A. A. Starobinsky, "Is there supernova evidence for dark energy metamorphosis?" Monthly 
Notices of the Royal Astronomical Society, vol. 354, no. 1, pp. 275291, 2004.

[15] T. R. Choudhury and T. Padmanabhan, "Cosmological parameters from supernova observations: a critical comparison of three data sets," Astronomy and Astrophysics, vol. 429, no. 3, pp. 807818, 2005.

[16] J. S. Alcaniz, "Testing dark energy beyond the cosmological constant barrier," Physical Review D, vol. 69, no. 8, Article ID 083521, 2004.

[17] H. J. Mosquera Cuesta, J. M. Salim, and M. Novello, "Cosmological redshift and nonlinear electrodynamics propagation of photons from distant sources," http://arxiv.org/abs/0710.5188.

[18] L. Campanelli, P. Cea, G. L. Fogli, and L. Tedesco, "Inflationproduced magnetic fields in nonlinear electrodynamics," Physical Review D, vol. 77, no. 4, Article ID 043001, 2008.

[19] L. Campanelli, P. Cea, G. L. Fogli, and L. Tedesco, "Inflationproduced magnetic fields in and models," Physical Review D, vol. 77, Article ID 123002, 2008.

[20] H. J. Mosquera Cuesta and J. M. Salim, "Non-linear electrodynamics and the gravitational redshift of highly magnetized neutron stars," Monthly Notices of the Royal Astronomical Society, vol. 354, no. 4, pp. L55-L59, 2004.

[21] H. J. M. Cuesta and J. M. Salim, "Nonlinear electrodynamics and the surface redshift of pulsars," Astrophysical Journal Letters, vol. 608, no. 2, pp. 925-929, 2004.

[22] H. J. Mosquera Cuesta, J. M. Salim, and J. A. De Freitas Pacheco, "Einstein's gravitational lensing and nonlinear electrodynamics," International Journal of Modern Physics A, vol. 21, no. 1, pp. 43-55, 2006.

[23] J. P. Mbelek, H. J. M. Cuesta, M. Novello, and J. M. Salim, "Nonlinear electrodynamics and the Pioneer 10/11 spacecraft anomaly, Europhysics Letters, vol. 77, no. 1, Article ID 19001, 2007.

[24] N. Riazi, H. Niad, and S. H. Hendi, "Black holes and solitons of the nonlinear isovector model," International Journal of Modern Physics A, vol. 21, no. 21, pp. 4343-4354, 2006.

[25] J. P. Mbelek and H. J. Mosquera Cuesta, "Non-linear electrodynamics and the variation of the fine structure constant," Monthly Notices of the Royal Astronomical Society, vol. 389, no. 1, pp. 199204, 2008.

[26] M. Marklund and P. K. Shukla, "Nonlinear collective effects in photon-photon and photon-plasma interactions," Reviews of Modern Physics, vol. 78, p. 591, 2006.

[27] J. Lundin, G. Brodin, and M. Marklund, "Short wavelength quantum electrodynamical correction to cold plasma-wave propagation," Physics of Plasmas, vol. 13, no. 10, Article ID 102102,2006

[28] E. Lundstrom, G. Brodin, J. Lundin et al., "Using high-power lasers for detection of elastic photon-photon scattering," Physical Review Letters, vol. 96, Article ID 083602, 2006.

[29] M. Born and L. Infeld, "Foundations of the new field theory," Proceedings of the Royal Society of London, vol. 144, p. 425, 1934.

[30] B. Hoffmann, "Gravitational and electromagnetic mass in the born-infeld electrodynamics," Physical Review, vol. 47, p. 877, 1935.

[31] M. Demiański, "Static electromagnetic geon," Foundations of Physics, vol. 16, no. 2, pp. 187-190, 1986.

[32] H. P. de Oliveira, "Non-linear charged black holes," Classical and Quantum Gravity, vol. 11, no. 6, p. 1469, 1994.

[33] G. W. Gibbons and D. A. Rasheed, "Electric-magnetic duality rotations in non-linear electrodynamics," Nuclear Physics B, vol. 454, no. 1-2, pp. 185-206, 1995.
[34] R.-G. Cai, D.-W. Pang, and A. Wang, "Born-Infeld black holes in (A)dS spaces," Physical Review D, vol. 70, no. 12, Article ID 124034, 2004.

[35] T. Kumar Dey, "Born-Infeld black holes in the presence of a cosmological constant," Physics Letters B, vol. 595, no. 1-4, pp. 484-490, 2004.

[36] M. H. Dehghani and H. R. R. Sedehi, "Thermodynamics of rotating black branes in $(\mathrm{n}+1)$-dimensional Einstein-BornInfeld gravity," Physical Review D, vol. 74, no. 12, Article ID 124018, 2006.

[37] M. H. Dehghani and S. H. Hendi, “Thermodynamics of rotating black branes in Gauss-Bonnet-Born-Infeld gravity," International Journal of Modern Physics D, vol. 16, no. 11, pp. 1829-1843, 2007.

[38] M. H. Dehghani, S. H. Hendi, A. Sheykhi, and H. Rastegar Sedehi, "Thermodynamics of rotating black branes in Einstein-Born-Infeld-dilaton gravity," Journal of Cosmology and Astroparticle Physics, no. 2, article 020, 2007.

[39] M. H. Dehghani, N. Alinejadi, and S. H. Hendi, "Topological black holes in Lovelock-Born-Infeld gravity," Physical Review D, vol. 77, no. 10, Article ID 104025, 2008.

[40] S. H. Hendi, "Rotating black branes in Brans-Dicke-Born-Infeld theory," Journal of Mathematical Physics, vol. 49, no. 8, Article ID 082501, 2008.

[41] M. Hassaïne and C. Martínez, "Higher-dimensional black holes with a conformally invariant Maxwell source," Physical Review $D$, vol. 75, no. 2, Article ID 027502, 2007.

[42] M. Hassaïne and C. Martínez, "Higher-dimensional charged black hole solutions with a nonlinear electrodynamics source," Classical and Quantum Gravity, vol. 25, no. 19, Article ID 195023, 2008.

[43] S. H. Hendi and H. R. Rastegar-Sedehi, "Ricci flat rotating black branes with a conformally invariant Maxwell source," General Relativity and Gravitation, vol. 41, no. 6, pp. 1355-1366, 2009.

[44] S. H. Hendi, "Topological black holes in Gauss-Bonnet gravity with conformally invariant Maxwell source," Physics Letters B, vol. 677, no. 3-4, pp. 123-132, 2009.

[45] H. Maeda, M. Hassaine, and C. Martinez, "Lovelock black holes with a nonlinear Maxwell field," Physical Review D, vol. 79, Article ID 044012, 2009.

[46] S. H. Hendi and B. E. Panah, "Thermodynamics of rotating black branes in Gauss-Bonnet-nonlinear Maxwell gravity," Physics Letters B, vol. 684, no. 2-3, pp. 77-84, 2010.

[47] S. H. Hendi, "The relation between F (R) gravity and Einsteinconformally invariant Maxwell source," Physics Letters B, vol. 690, no. 3, pp. 220-223, 2010.

[48] S. H. Hendi, "Slowly rotating black holes in einstein-generalized maxwell gravity," Progress of Theoretical Physics, vol. 124, no. 3, pp. 493-502, 2010.

[49] S. H. Hendi, "Rotating black branes in the presence of nonlinear electromagnetic field," European Physical Journal C, vol. 69, no. 1, pp. 281-288, 2010.

[50] S. H. Hendi, "Rotating black string with nonlinear source," Physical Review D, vol. 82, Article ID 064040, 2010.

[51] S. H. Hendi, S. Kordestani, and S. N. Doosti Motlagh, "The effects of nonlinear Maxwell source on the magnetic solutions in Einstein-Gauss-Bonnet gravity," Progress of Theoretical Physics, vol. 124, no. 6, pp. 1067-1082, 2010.

[52] S. H. Hendi, "Charged BTZ-like black holes in higher dimensions," The European Physical Journal C, vol. 71, p. 1551, 2011. 
[53] S. H. Hendi, "Asymptotic charged BTZ black hole solutions," Journal of High Energy Physics, no. 3, article 065, 2012.

[54] S. H. Hendi, "Asymptotic Reissner-Nordström black holes," Annals of Physics, vol. 333, pp. 282-289, 2013.

[55] S. H. Hendi and A. Sheykhi, "Charged rotating black string in gravitating nonlinear electromagnetic fields," Physical Review $D$, vol. 88, Article ID 044044, 2013.

[56] D. H. Delphenich, "Nonlinear electrodynamics and QED," http://arxiv.org/abs/hep-th/0309108.

[57] D. H. Delphenich, "Nonlinear optical analogies in quantum electrodynamics," http://arxiv.org/abs/hep-th/0610088.

[58] M. G. Richarte, "Cylindrical wormholes in DGP gravity," Physical Review D, vol. 87, Article ID 067503, 2013.

[59] S. Kar and D. Sahdev, "Evolving Lorentzian wormholes," Physical Review D, vol. 53, no. 2, pp. 722-730, 1996.

[60] M. Cataldo, P. Salgado, and P. Minning, "Self-dual Lorentzian wormholes in $n$-dimensional Einstein gravity," Physical Review $D$, vol. 66, no. 12, Article ID 124008, 2002.

[61] A. DeBenedictis and A. Das, "Higher dimensional wormhole geometries with compact dimensions," Nuclear Physics B, vol. 653, no. 1-2, pp. 279-304, 2003.

[62] B. Bhawal and S. Kar, "Lorentzian wormholes in EinsteinGauss-Bonnet theory," Physical Review D, vol. 46, no. 6, pp. 2464-2468, 1992.

[63] M. H. Dehghani and S. H. Hendi, "Wormhole solutions in Gauss-Bonnet-Born-Infeld gravity," General Relativity and Gravitation, vol. 41, no. 8, pp. 1853-1863, 2009.

[64] S. H. Hendi, "The effects of conformally invariant Maxwell source on the magnetic wormhole solutions in Gauss-Bonnet gravity," Canadian Journal of Physics, vol. 89, pp. 281-287, 2011.

[65] K. Ghoroku and T. Soma, "Lorentzian wormholes in higherderivative gravity and the weak energy condition," Physical Review D, vol. 46, no. 4, pp. 1507-1516, 1992.

[66] S. H. Hendi, "Magnetic wormhole solutions in Einstein-GaussBonnet gravity with power Maxwell invariant source," Journal of Mathematical Physics, vol. 52, no. 4, Article ID 042502, 2011.

[67] M. Visser, Lorentzian Wormholes, AIP Press, Woodbury, NY, USA, 1996.

[68] F. S. N. Lobo and P. Crawford, "Linearized stability analysis of thin-shell wormholes with a cosmological constant," Classical and Quantum Gravity, vol. 21, no. 2, pp. 391-404, 2004.

[69] E. F. Eiroa and G. E. Romero, "Linearized stability of charged thin-shell wormholes," General Relativity and Gravitation, vol. 36, no. 4, pp. 651-659, 2004.

[70] J. P. S. Lemos and F. S. N. Lobo, "Plane symmetric traversable wormholes in an anti-de Sitter background," Physical Review D, vol. 69, no. 10, Article ID 104007, 2004.

[71] M. G. Richarte and C. Simeone, "Traversable wormholes in a string cloud," International Journal of Modern Physics D, vol. 17, no. 8, pp. 1179-1196, 2008.

[72] M. G. Richarte and C. Simeone, "Thin-shell wormholes supported by ordinary matter in Einstein-Gauss-Bonnet gravity," Physical Review D, vol. 76, Article ID 087502, 2007, Erratum in Physical Review D, vol. 77, Article ID 089903, 2008.

[73] M. G. Richarte and C. Simeone, "Wormholes in Einstein-BornInfeld theory," Physical Review D, vol. 80, Article ID 104033, 2009, Erratum in Physical Review D, vol. 81, Article ID 109903 , 2010.

[74] E. F. Eiroa, M. G. Richarte, and C. Simeone, "Thin-shell wormholes in Brans-Dicke gravity," Physics Letters A, vol. 373, no. 1, pp. 1-4, 2008, Erratum in Physics Letters A, vol. 373, p. 2399, 2008.

[75] G. A. S. Dias and J. P. S. Lemos, "Thin-shell wormholes in $d$-dimensional general relativity: solutions, properties, and stability," Physical Review D, vol. 82, Article ID 084023, 2010.

[76] S. H. Mazharimousavi, M. Halilsoy, and Z. Amirabi, "Stability of thin-shell wormholes supported by normal matter in einsteinmaxwell-gauss-bonnet gravity," Physical Review D, vol. 81, no. 10, Article ID 104002, 2010.

[77] M. H. Dehghani and M. R. Mehdizadeh, "Lovelock thin-shell wormholes," Physical Review D, vol. 85, no. 2, Article ID 024024, 2012.

[78] P. Kanti, B. Kleihaus, and J. Kunz, "Stable Lorentzian wormholes in dilatonic Einstein-Gauss-Bonnet theory," Physical Review D, vol. 85, no. 4, Article ID 044007, 2012.

[79] I. Bochicchio and E. Laserra, "Intra-Galactic thin shell wormhole and its stability," http://arxiv-web3.library.cornell .edu/abs/1301.1532.

[80] F. Rahaman, A. Banerjee, and I. Radinschi, "A new class of stable $(2+1)$ dimensional thin shell Wormhole," International Journal of Theoretical Physics, vol. 51, no. 6, pp. 1680-1691, 2012.

[81] A. A. Usmani, Z. Hasan, F. Rahaman, S. A. Rakib, S. Ray, and P. K. F. Kuhfittig, "Thin-shell wormholes from charged black holes in generalized dilaton-axion gravity," General Relativity and Gravitation, vol. 42, no. 12, pp. 2901-2912, 2010.

[82] J. P. S. Lemos and F. S. N. Lobo, "Plane symmetric thin-shell wormholes: solutions and stability," Physical Review D, vol. 78, no. 4, Article ID 044030, 2008.

[83] K. A. Bronnikov and A. A. Starobinsky, "Once again on thin-shell wormholes in scalar-tensor gravity," Modern Physics Letters A, vol. 24, p. 1559, 2009.

[84] S. H. Hendi, "Magnetic branes supported by a nonlinear electromagnetic field," Classical and Quantum Gravity, vol. 26, no. 22, Article ID 225014, 2009.

[85] M. Visser, "Traversable wormholes: some simple examples," Physical Review D, vol. 39, no. 10, pp. 3182-3184, 1989.

[86] F. Rahaman, S. Chakraborty, and M. Kalam, "Thin-shell wormhole in the heterotic string theory," International Journal of Modern Physics D, vol. 16, no. 10, pp. 1669-1681, 2007.

[87] P. K. F. Kuhfittig, "Theoretical construction of stable traversable wormholes," Central European Journal of Physics, vol. 8, pp. 364368, 2010.

[88] P. E. Kashargin and S. V. Sushkov, "Rotating thin-shell wormhole from glued Kerr spacetimes," Gravitation and Cosmology, vol. 17, no. 2, pp. 119-125, 2011.

[89] M. Sharif and M. Azam, "Stability analysis of thin-shell wormholes from charged black string," Journal of Cosmology and Astroparticle Physics, vol. 2013, no. 04, article 023, 2013.

[90] M. Sharif and M. Azam, "Spherical thin-shell wormholes and modified Chaplygin gas," Journal of Cosmology and Astroparticle Physics, vol. 2013, no. 05, article 025, 2013.

[91] F. S. N. Lobo and P. Crawford, "Stability analysis of dynamic thin shells," Classical and Quantum Gravity, vol. 22, pp. 4869-4886, 2005.

[92] J. Stachel, "Globally stationary but locally static space-times: a gravitational analog of the Aharonov-Bohm effect," Physical Review D, vol. 26, no. 6, pp. 1281-1290, 1982.

[93] J. Maldacena, "The large $N$ limit of superconformal field theories and supergravity," Advances in Theoretical and Mathematical Physics, vol. 2, no. 2, pp. 231-252, 1998. 
[94] E. Witten, "Anti de Sitter space and holography," Advances in Theoretical and Mathematical Physics, vol. 2, no. 2, pp. 253-291, 1998.

[95] O. Aharony, S. S. Gubser, J. Maldacena, H. Ooguri, and Y. $\mathrm{Oz}$, "Large $N$ field theories, string theory and gravity," Physics Reports, vol. 323, no. 3-4, pp. 183-386, 2000. 

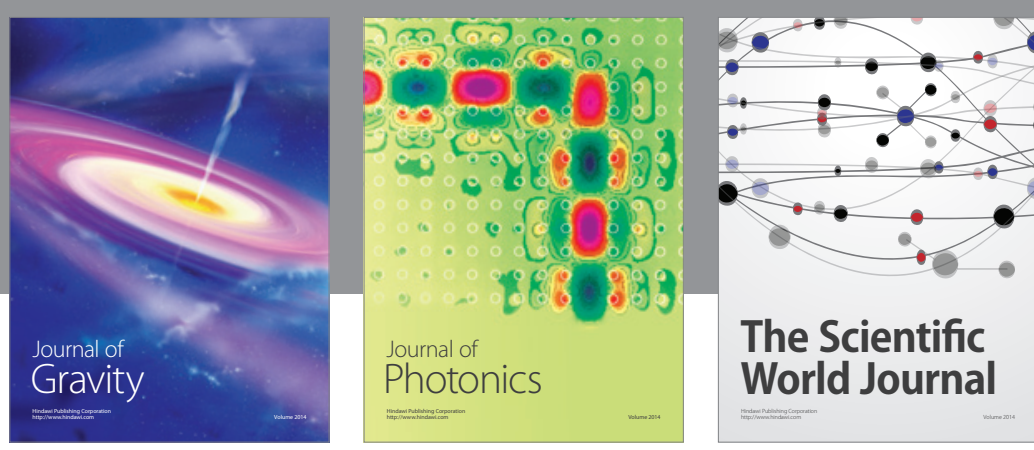

The Scientific World Journal
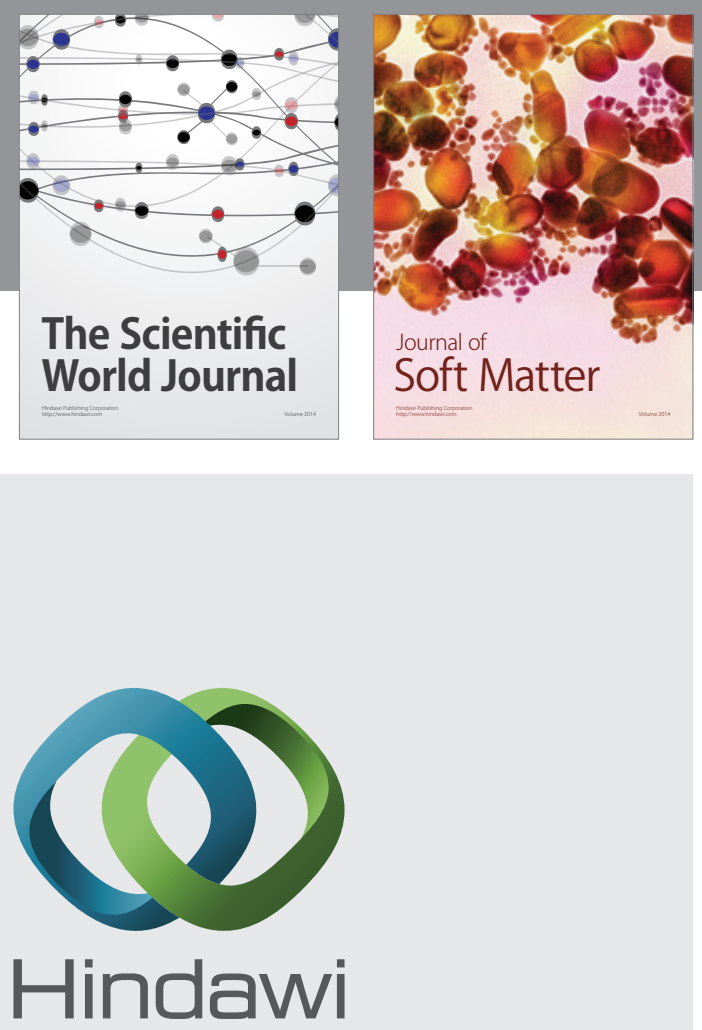

Submit your manuscripts at

http://www.hindawi.com

nternational Journal of

Statistical Mechanics
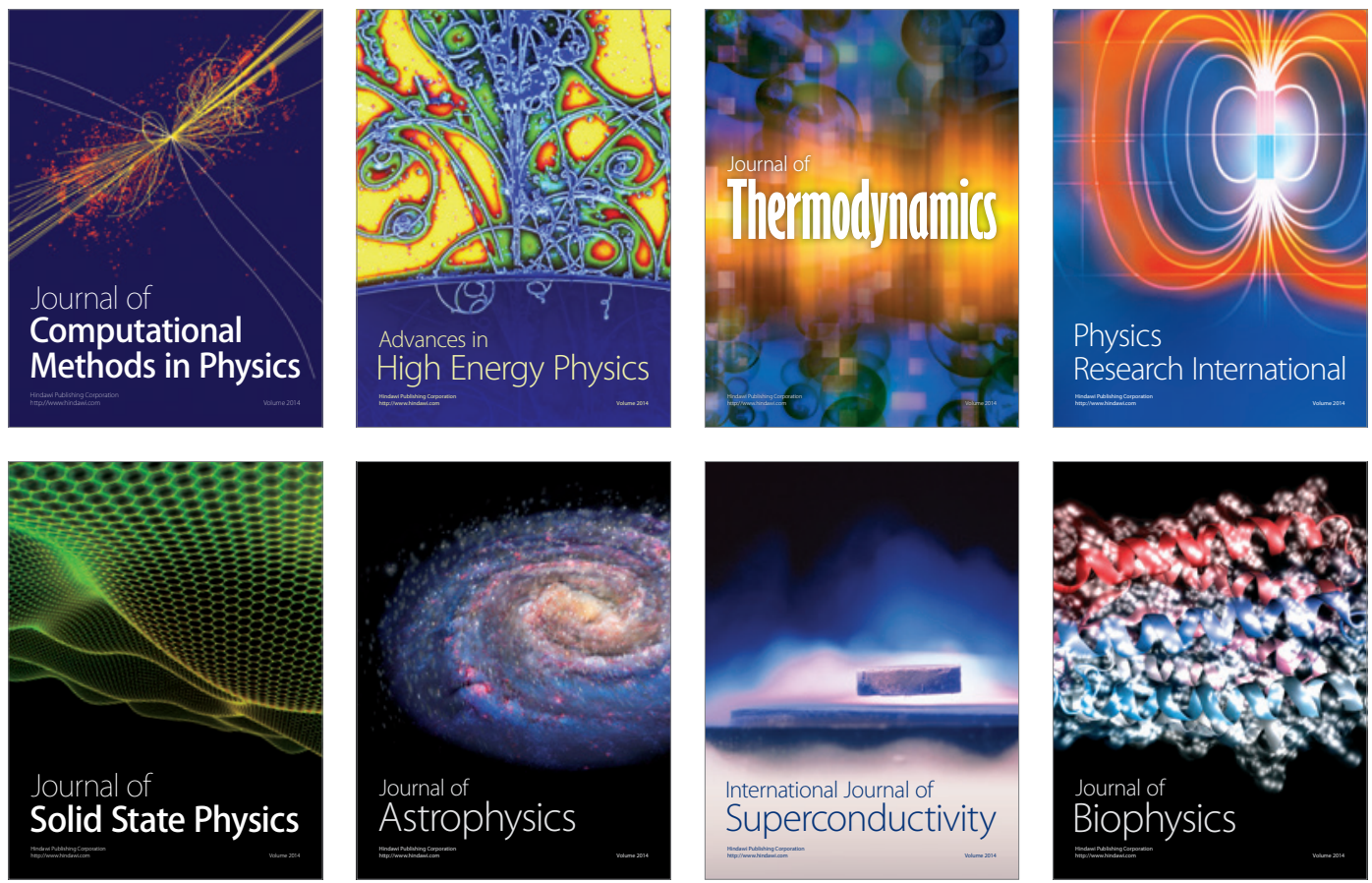
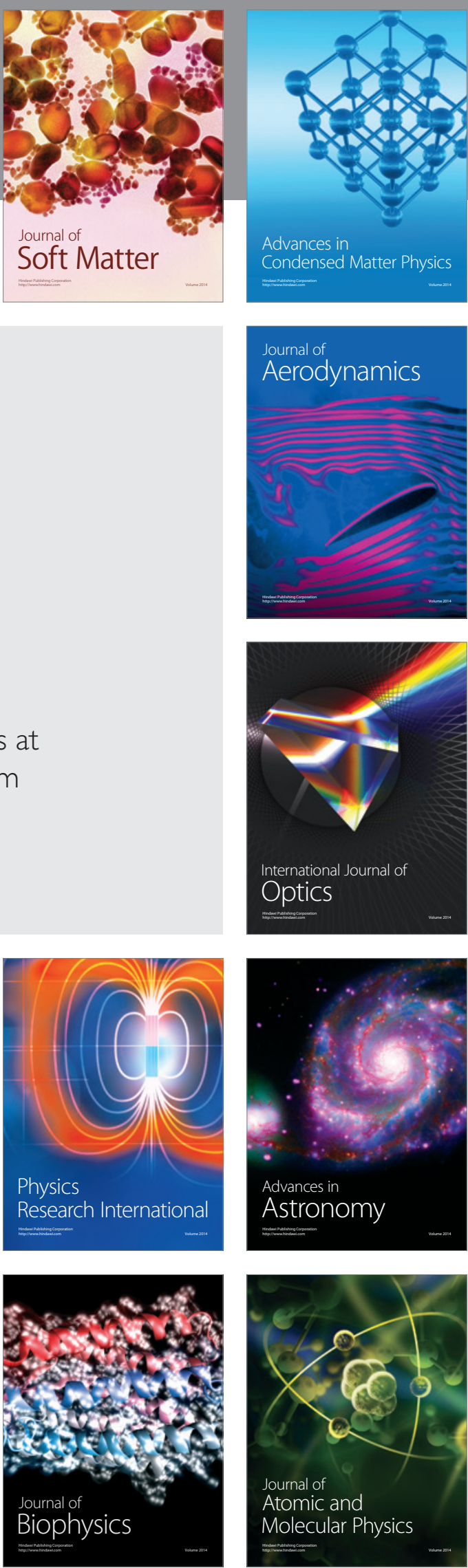